\title{
Estimativa dos Perfis de Dormentes em Pontes sem Lastro Utilizando Sensor de Perfil Laser ${ }^{\star}$
}

\author{
Jorge Ruan Zeferino Conti* Diego Santana Conceição* \\ Mariana Rampinelli Fernandes * Fabricio Bortolini de Sá * \\ Flávio Garcia Pereira* André Stanzani Franca** \\ Luciano de Sousa Pereira ${ }^{* *}$ Maria Rachel Cardoso Máximo** \\ * Instituto Federal de Educação, Ciência e Tecnologia do Espírito \\ Santo - IFES. \\ (e-mail: jorgeconti@live.com,diegosantana011@gmail.com, \\ mariana.rampinelli@ifes.edu.br,fabricio.sa@ifes.edu.br, \\ flavio.garcia@ifes.edu.br). \\ ** Vale S. A. (e-mail:andre.franca@vale.com.br, \\ luciano.pereira@vale.com,rachel.maximo@vale.com).
}

\begin{abstract}
This work deals with the $3 \mathrm{D}$ reconstruction of a non-ballast bridge sleepers in a railway problem by using a profile laser sensor. In order to perform this reconstruction, the laser is installed in a mobile platform and scans the entire bridge structure. The data provided by the laser were processed and a 3D point cloud was created. From this point cloud, some images are generated and by processing them the information of the bridge structure is obtained. In this work, a non-ballast bridge model was used and the proposed method was able to perform the rebuilding of the sleepers and estimate the carves that they must have to fit in the girders and bracings of the non-ballast bridge. The percentage error between the model and the rebuilt structure was approximately $1.5 \%$. It is expected the reduction of this error when the method is applied at real size bridge.

Resumo: Este trabalho aborda o problema da reconstrução 3D dos dormentes de uma ponte sem lastro em ferrovias utilizando um sensor de perfil laser. Para realizar essa reconstrução, o laser foi instalado em uma plataforma móvel e faz a varredura de toda a estrutura da ponte. Os dados obtidos pelo laser foram processados e uma nuvem de pontos 3D foi criada. A partir dessa nuvem, são geradas imagens e com elas obtidas as informações da estrutura da ponte. Neste trabalho foi utilizada uma maquete de uma ponte sem lastro e o método proposto foi capaz de realizar a reconstrução dos dormentes e estimar os entalhes que os mesmos devem possuir para se encaixarem nas longarinas e contraventamentos da ponte sem lastro. $\mathrm{O}$ erro percentual entre a maquete e a estrutura reconstruída foi de aproximadamente 1,5\%. Espera-se a redução desse erro quando o método for aplicado em uma ponte de tamanho real.
\end{abstract}

Keywords: Profile Laser Sensor; 3D Reconstruction;.Pontes sem Lastro; Dormentes.

Palavras-chaves: Sensor de Perfil Laser; Reconstrução 3D; Pontes sem Lastro; Dormentes.

\section{INTRODUÇÃO}

O sistema ferroviário teve origem no final do século XVIII e início do século XIX, na Inglaterra, no auge da Revolução Industrial. No Brasil, as condições econômicas e sociais que demandassem a construção de ferrovias surgiram somente na segunda metade do século XIX. Durante a Segunda Guerra Mundial, com o crescimento exponencial da demanda por minério de ferro, a Estrada de Ferro Vitória a Minas (EFVM) teve a sua importância consolidada no cenário nacional.

A ferrovia é um dos meios de transporte mais eficientes para longas distâncias e predominantemente utilizado pela

* Os autores gostariam de agradecer à Vale S. A. pelo suporte financeiro para o desenvolvimento deste trabalho. indústria de mineração e na movimentação de granéis sólidos. Ela é composta pelos ativos de material rodante (locomotivas e vagões, entre outros) e os ativos da via permanente Franca (2017).

A via permanente é formada pelos elementos da superestrutura e da infraestrutura. A infraestrutura é composta pelo conjunto das obras de arte (pontes e viadutos) e de terraplenagem, seja com cortes ou aterros, desde o subleito, passando pela base até a camada de sublastro. Já a superestrutura ferroviária é composta pelos dormentes, trilhos, conjuntos de fixações dos trilhos aos dormentes e pela camada de lastro (Silva, 2002; Franca, 2017).

A via permanente é construída de modo a ser renovada, quando o desgaste dos seus componentes atingir o limite de tolerância exigido pela segurança (Lourenço, 2014). A 
manutenção do sistema ferroviário apresenta um custo significativo tendo em vista da reposição de trilhos e acessórios metálicos ao longo do tempo, como também da troca de dormentes de forma periódica. Por isso, fazse necessária a implementação de tecnologias que possam ser aplicadas em conjunto com os métodos já existentes para avaliar o estado do sistema ferroviário ou efetuar a manutenção do mesmo (Brochado and Pires, 2007).

O trabalho apresentado neste artigo tem os dormentes como objetos de estudo. Mais especificamente, os dormentes de madeira que compõem a estrutura das pontes sem lastro. Nessas pontes, as longarinas são os componentes responsáveis pela sustentação dos dormentes. A Figura 1 (a) apresenta a vista superior de como é feita a sustentação dos dormentes em uma ponte sem lastro, enquanto a vista inferior está representada na Figura 1 (b), onde podem ser vistas as logarinas de sustentação e os contraventamentos.

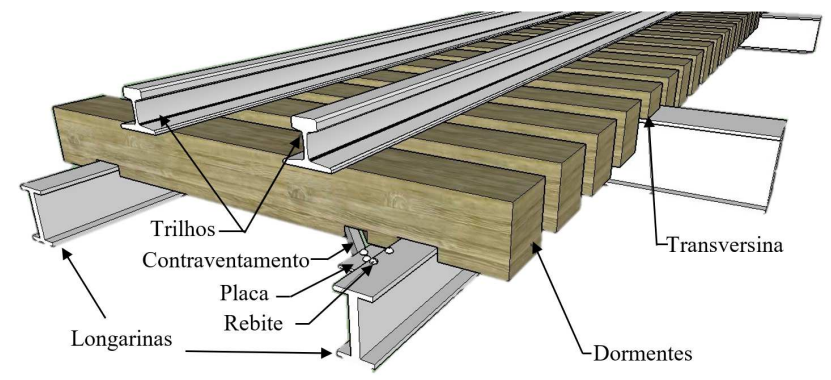

(a)

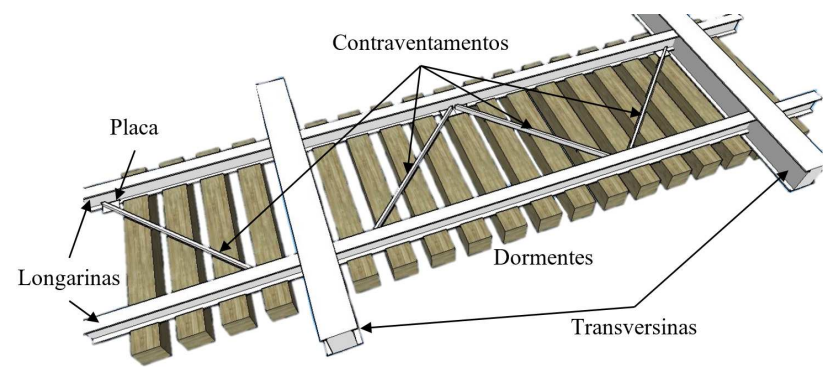

(b)

Figura 1. Estrutura superior (a) e inferior (b) de uma ponte sem lastro.

O dormente é um dos elementos mais importantes da superestrutura ferroviária (Campos, 2015). Ele pode ser de madeira, metal, concreto ou material polimérico. Os dormentes de madeira apresentam como principal qualidade a flexibilidade, o que lhes conferem a capacidade de suportar vibrações das ações dinâmicas atuantes na via permanente (Campos, 2015).

Apesar das vantagens dos dormentes de madeira, os mesmos devem ser substituídos quando ocorre a degradação por apodrecimento ou o comprometimento da capacidade de suportar cargas verticais, de manter o nivelamento da via e o poder de retenção das fixações. Os dormentes sofrem deformação permanente em virtude das tensões exercidas sobre a ferrovia, assim quando os mesmos necessitam ser substituídos, devem ter suas dimensões apuradas. Esse processo demanda tempo, mão de obra e equipamentos para medição (Vale, 2009).
O trabalho de entalhe que os novos dormentes deve apresentar, garantindo a não interação destes com estruturas específicas da ponte sem lastro, são executadas na própria ponte. Submetendo um grupo de funcionários a riscos operacionais de trabalho com movimentação de cargas pesadas em altura. Isso porque, com o passar dos anos ocorrem acomodações estruturais, alterações de projeto e ajustes. Assim, no momento do assentamento de novos dormentes, o especialista deve marcar, girar e entalhar os dormentes enquanto se equilibra sobre a ponte vazada. Isso toma muito tempo e expõe o trabalhador a riscos de acidentes. Daí a necessidade de se desenvolver uma ferramenta que possa auxiliar nessa substituição dos dormentes em ponte sem lastro.

Com o intuito de reduzir o tempo de parada da linha e, ao mesmo tempo, reduzir os riscos operacionais da substituição dos dormentes de uma ponte sem lastro, este trabalho propõe a reconstrução tridimensional da estrutura, em escala, de uma ponte sem lastro utilizando um sensor de perfil laser. Desse modo, será possível identificar as interações e intersecções dos dormentes com a estrutura da ponte.

Como os novos dormentes já estarão com os seus perfis definidos no momento da manutenção na ponte, o tempo de substituição dos mesmos será menor que atualmente. Assim, os especialistas que efetuam a troca dos dormentes estarão menos sujeitos a riscos operacionais, uma vez que os mesmos necessitarão de menos tempo para realizar tal tarefa.

Com o método de reconstrução apresentado neste trabalho, espera-se obter resultados semelhantes ao desenho apresentado na Figura 2, que mostra os entalhes que devem ser feitos em cada dormente de forma que eles se encaixem na estrutura de uma ponte sem lastro.

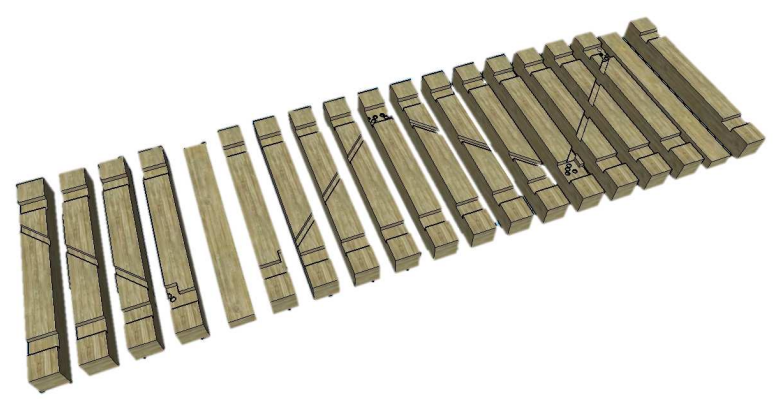

Figura 2. Entalhes dos dormentes de uma ponte sem lastro.

\section{REFERENCIAL TEÓRICO}

No trabalho desenvolvido por (Lubowiecka et al., 2009), os autores utilizaram um laser para realizar a reconstrução tridimensional de uma ponte antiga localizada na Espanha. Adicionalmente, foi utilizado também um radar de penetração no solo (GPR) para analisar internamente as estruturas da ponte. Bosché (2010) realizou um estudo para controle do andamento da construção de estrutura metálica de um edifício. Utilizando um sensor de varredura laser e algoritmos de reconhecimento de padrões, o autor demostrou que o sistema era capaz de reconstruir as estruturas metálicas de uma obra em andamento. 
A pesquisa apresentada por (Rabah et al., 2013), traz um método para detecção de fissuras e mapeamento automático em concretos e partir de dados obtidos por um sensor laser de varredura. Os resultados mostraram que a medição das fissuras utilizando o método proposto é tão preciso quanto os obtidos pelo método tradicional utilizando o equipamentos topográficos.

Diversos trabalhos foram desenvolvidos para realizar o mapeamento tridimensional de um ambiente, ou uma estrutura, utilizando um sensor laser de varredura bidimensional. Por exemplo, no trabalho de (Harrison and Newman, 2008), foi desenvolvido um sistema completo capaz de gerar uma nuvem tridimensional de pontos de alta qualidade utilizando um laser bidimensional acoplado a uma plataforma de movimento contínuo.

Os autores da pesquisa apresentada por Klimentjew et al. (2009) utilizaram um laser bidimensional acoplado a diferentes plataformas móveis para descrever dois métodos diferentes de reconstrução. Foram utilizados um robô manipulador e uma plataforma pan-tilt para realizar a reconstrução de objetos sob diferentes perspectivas. Ambos os métodos apresentaram resultados satisfatórios.

O trabalho de (Llamazares et al., 2012) utilizou um sensor laser bidimensional fixado a um robô móvel inclinado para baixo afim realizar uma reconstrução tridimensional do caminho percorrido pelo robô. Utilizando a nuvem de pontos capturadas o autor desenvolveu um método para minimizar o erro de odometria. Bons resultados foram alcançados em testes de campo aberto.

Os pesquisadores do trabalho apresentado por Ren et al. (2011) desenvolveram uma plataforma móvel específica para captura das deformações dos trilhos de vias de alta velocidade na China. Tal plataforma móvel possui um sistema de absorção de vibrações para minimizar erros intrínsecos do sistema de aquisição do laser acoplado à estrutura.

A pesquisa realizada por Yang and Fang (2014), destaca o aumento pela procura relacionadas sobre "fatores humanos" no final da década de 90, após alguns graves acidentes ocorridos por erros humanos, esta pesquisa destaca a importância de fornecer um ambiente de trabalho confortável e seguro, o qual pode ser obtido com a inserção de equipamentos que retire do operador a necessidade de esforço e exposição constante em locais de riscos.

As referências apresentadas demonstram a viabilidade da utilização de sensores laser para a reconstrução tridimensional de uma ponte ferroviária. A precisão das medidas proporcionadas por esse sensor promete bons resultados no mapeamento das estruturas.

\section{MATERIAIS E MÉTODOS}

Com o intuito de desenvolver um método capaz de realizar a reconstrução da estrutura dos dormentes de uma ponte sem lastro, foi gerada em uma impressora 3D, uma estrutura em miniatura das longarinas e dos dormentes apoiados sobre elas. Na Figura 3 pode-se ver o modelo em CAD utilizado para imprimir a estrutura dos dormentes e das longarinas.

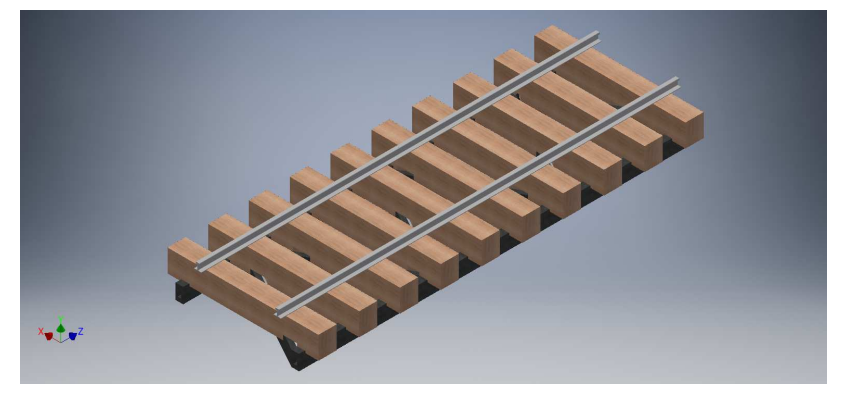

Figura 3. Modelo em CAD para impressão.

A maquete gerada a partir do desenho apresentado na Figura 3 pode ser vista nas Figuras 4 (a) e (b). A Figura 4 (a) mostra a vista superior da maquete e a Figura 4 (b) traz a representação da parte inferior dos dormentes com seus entalhes para que possam ser encaixados nas longarinas e nos contraventamentos.

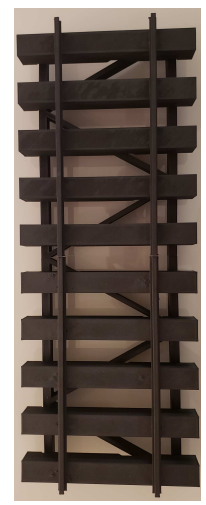

(a)

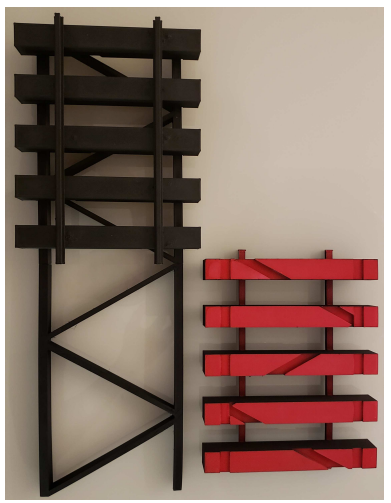

(b)
Figura 4. Ponte impressa na 3D.

O sensor utilizado neste trabalho para capturar os dados da maquete da ponte sem lastro impressa e, após isso, gerar um modelo tridimensional da mesma, inclusive dos entalhes existentes em seus dormentes, foi o sensor de perfil MLSL 276, fabricado pela Wenglor. Tal sensor possui alta resolução (270 a $1170 \mu \mathrm{m})$, e alta taxa de amostragem (200 a 4000 medidas por segundo). O sensor é constituído de duas partes fundamentais, o emissor de luz estruturada e a matriz CCD sensível a reflexão da luz.

O princípio de funcionamento do MLSL276 é dependente da linha estruturada gerada pelo emissor de luz e da matriz CCD, representada pelo número 3 na Figura 5. O número 4 representa o ângulo gerado pelo feixe de luz e o caminho de reflexão para a matriz CCD (que possui 1280 pixels na direção do eixo X), o reflexo da luz passa por um conjunto de lentes que antecedem a matriz CCD, responsáveis pelo seu redimensionamento incidente nos pontos sensitivos da matriz, desta maneira é possível obter informações de profundidade e abertura do feixe de luz a qual correspondem respectivamente às coordenadas $\mathrm{Z}$ e X capturadas pelas linhas e colunas de pixels da matriz.

O modelo de sensor em estudo possui uma área de trabalho bem definida, realçada em cinza na Figura 6. A área útil que é utilizada para realizar o levantamento de dados da ponte sem lastro, destacada com retângulo, apresenta extensão de 1 a 1,5 metros de profundidade na coordenada 


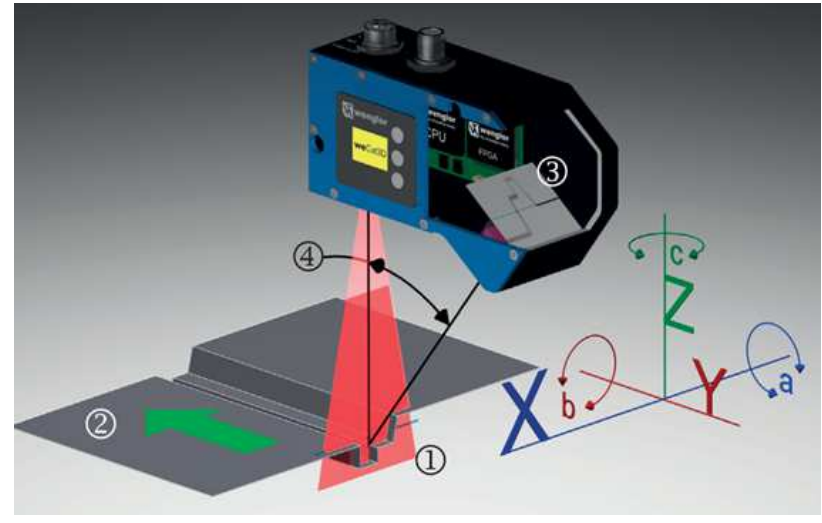

Figura 5. Princípio de funcionamento do sensor de perfil laser utilizado neste trabalho.

Z. E abertura de feixe entre 0,9 a 1,3 metros na orientação $\mathrm{X}$.

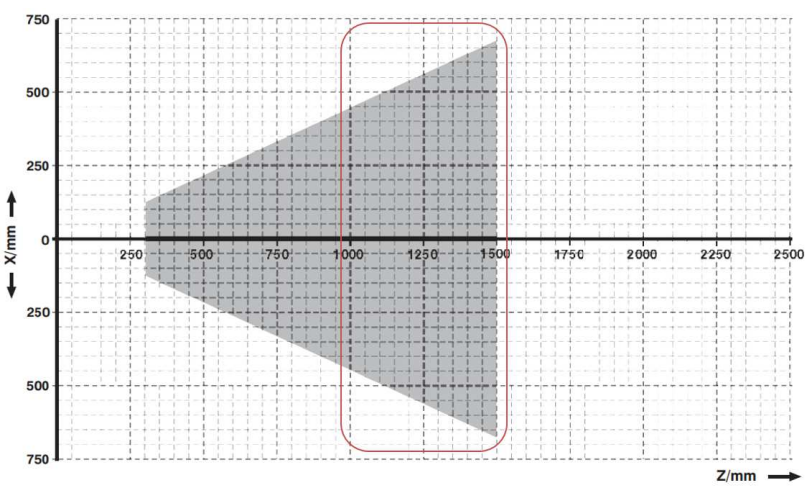

Figura 6. Área de trabalho do sensor MLSL276.

O suporte do projeto de ponte sem lastro foi projetado de tal maneira a posicionar os lasers a uma distância que varia de 1,3 a 1,4 metros (na coordenada $Z$ do laser) da superfície das longarinas da ponte sem lastro, como pode ser observado na Figura 7, conseguindo assim utilizar a zona com maior abertura do sensor, utilizando a faixa de 1,1 à 1,3 metros (na coordenada $\mathrm{X}$ do laser).

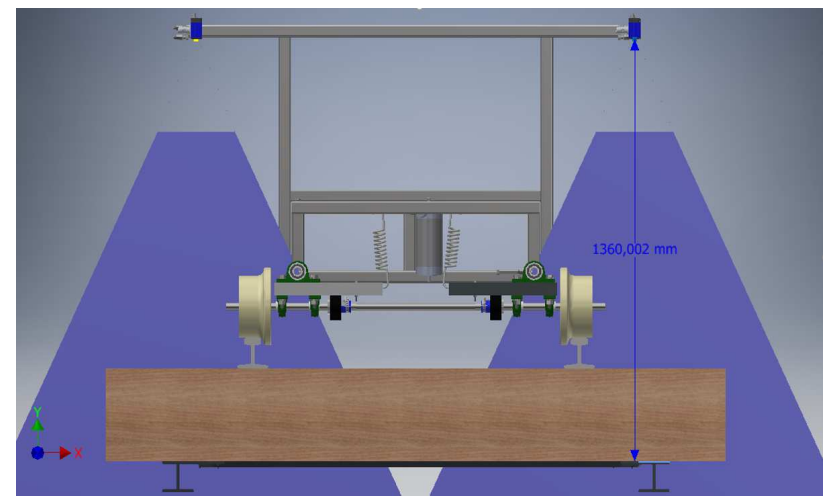

Figura 7. Vista frontal do suporte dos lasers de ponte sem lastro.

\subsection{Desenvolvimento}

O método para reconstrução tridimensional de estruturas de pontes sem lastro proposto neste trabalho é dividido em 4 etapas: i) a Captura dos Dados, ii) a Conversão dos Dados para Imagem, iii) Obtenção da Nova Dormentação e iv) Obtenção do Modelo 3D. A Figura 8 mostra as etapas do projeto proposto neste trabalho.

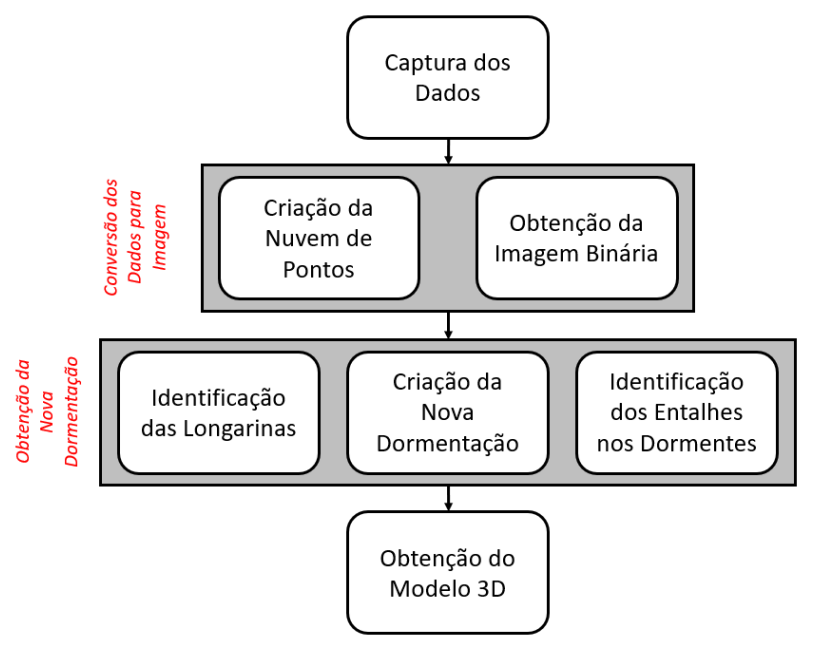

Figura 8. Etapas de processamento de dados.

\section{i) Captura dos Dados}

Esta etapa consiste na aquisição de pontos gerados pelo sensor de perfil laser durante o escaneamento, gerar um arquivo contendo os valor de distâncias tridimensional para cada ponto em um sistema de coordenadas inerciais, com posterior interpretação pelo software desenvolvido.

ii) Conversão dos Dados para Imagem

Nesta etapa do projeto o arquivo contendo so dados da estrutura da ponte é plotado gerando uma nuvem de pontos, que pode ser vista na Figura 9. O movimento da plataforma foi feito de maneira que os dormentes estariam na posição horizontal e as longarinas na vertical. Existe a presenta de alguns pontos na frente da ponte que representa o cabeamento do sensor, optou-se por utilizar esses dados para demostrar a robustez do projeto com pontos fora da área de interesse.

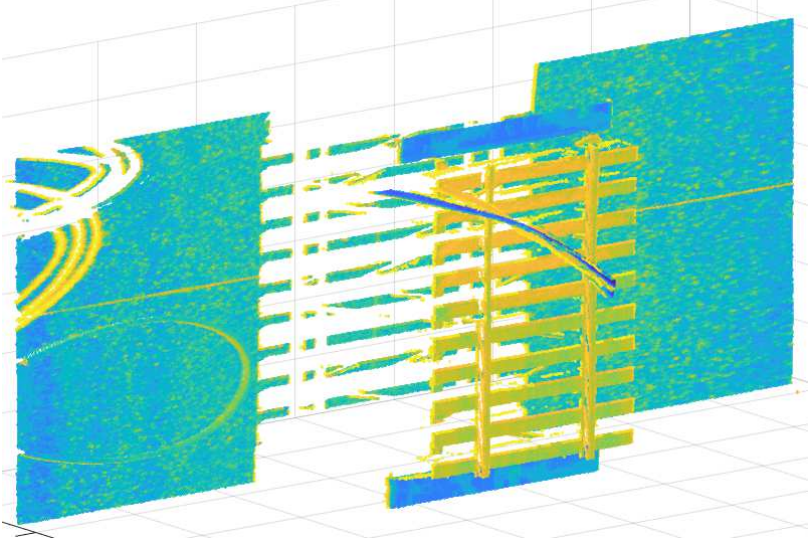

Figura 9. Nuvem com todos pontos.

Após obter esses pontos, gera-se uma imagem binária contendo apenas informações de localização das longarinas e dos contraventamentos da estrutura da ponte. Essa 
imagem é gerada encontrando os pontos dentro de determinados valores de profundidade (coordenada Z do sensor). A Figura 10 traz a imagem binarizada referente à nuvem de pontos apresentada na Figura 9.

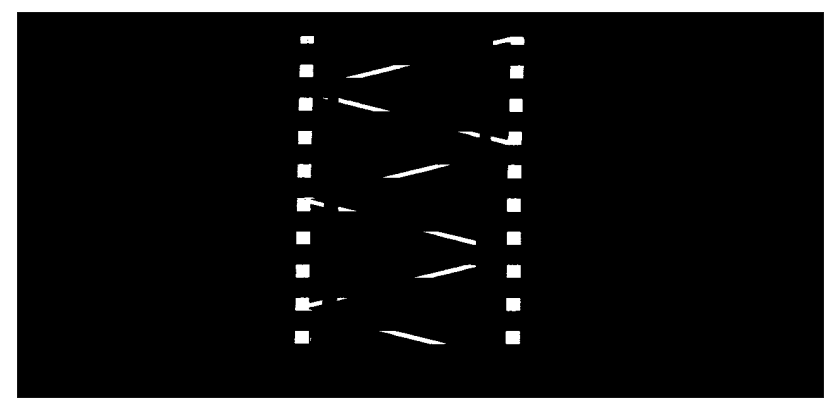

Figura 10. Imagem binarizada.

\section{iii) Obtenção da Nova Dormentação}

Após a obtenção da imagem binária inicia-se o processo de busca pelas longarinas e pelos contraventamentos da estrutura da ponte. As longarinas aparecem verticalmente na imagem binarizada e, além disso são representadas pelos conjuntos de blobs ao lado esquerdo e direito nessa mesma imagem. Na Figura 11 é possível observar o histograma das colunas da imagem binária. Os picos identificados pelos histogramas ilustram a localização das longarinas já destacadas em linha pontilhada azul.

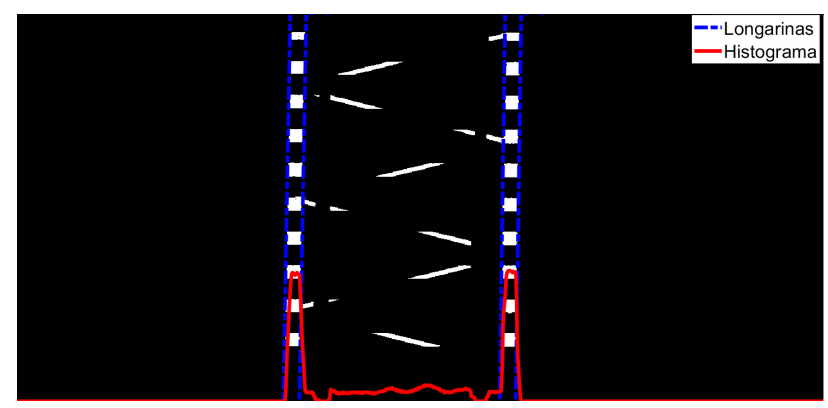

Figura 11. Histograma (vermelho) e Longarinas (azul).

Para localizar os contraventamentos, faz-se a exclusão dos blobs pertencentes às longarinas e utiliza-se o restante da imagem. Dessa maneira, todos os blobs presentes nesse espaço são classificados como contraventamentos. Dá-se início, então, ao diagnóstico individual buscando por blobs vizinhos que possam pertencer aos mesmos contraventamentos. Após a classificação de cada blob com o seu respectivo contraventamento, é determinada a reta comum que os une. Nos casos onde apenas um blob é identificado como contraventamento, utiliza-se o maior eixo da elipse circunscrita ao blob. A Figura 12 apresenta os resultados obtidos para as longarinas (em azul) e para os contraventamentos (em vermelho e verde).

Nesta etapa toda a parte estrutural da ponte já foi obtida. É preciso agora, realizar o posicionamento dos dormentes sobre a estrutura e, assim, estimar os entalhes que devem ser feitos nos mesmos. Em uma ponte sem lastro, os dormentes estão posicionados sobre as longarinas e, durante o processo de captura dos dados, impedem que tais longarinas sejam totalmente detectadas pelo sensor. Logo, os dormentes estão posicionados nos espaços vazios

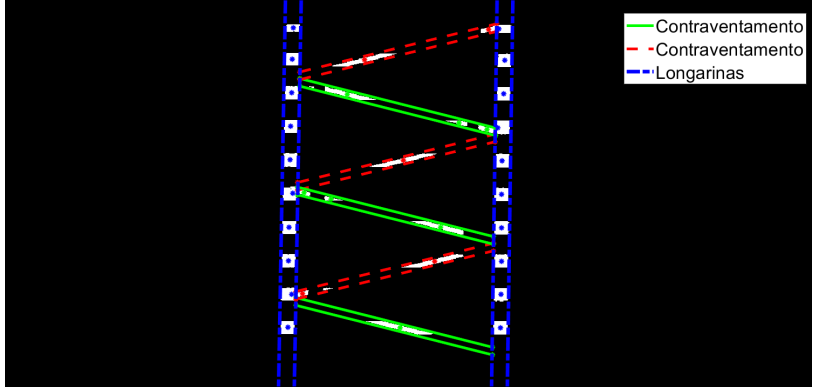

Figura 12. Longarinas (azul) e contraventamentos (verde e vermelho).

entre os blobs destacados pelas linhas azuis na Figura 12, que representam as longarinas. A Figura 13 apresenta o resultado da detecção das longarinas e do posicionamento dos dormentes.

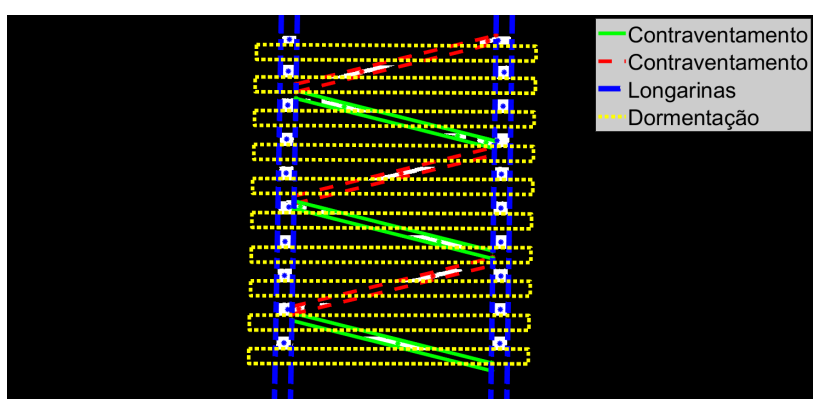

Figura 13. Longarinas e posicionamento dos dormentes.

Após estimar as posições das longarinas, contraventamentos e também a localização dos novos dormentes sobre essas estruturas, é feito o mapeamento dos entalhes nos dormentes para que estes posteriormente possam ser assentados na ponte sem lastro. Os entalhes dos novos dormentes são obtidos extraindo as partes estruturais da ponte presente no interior de cada dormente da Figura 13.

A Figura 14 ilustra o resultado obtido desta etapa. Os dormentes foram destacados com linhas pontilhadas na cor preta. Cada dormente possui entalhes especifico no qual os diferem dos demais, os traço pontilhados destacados em azul pertencem às longarinas, esse tipo de entalhe é realizado com meia polegada de profundidade, enquanto os contraventamentos que estão destacados de verde e vermelho, são feitos com uma polegada e meia. A cor verde foi usada para realçar os encontros de contraventamentos com longarinas existentes no entalhe do dormente.

iv) Obtenção do Modelo 3D

A última etapa do método apresentado neste trabalho consiste em utilizar as informações já obtidas nos passos anteriores e gerar um modelo tridimensional dos dormentes com os seus respectivos entalhes. Para isso, é feita inicialmente a transformação das informações obtidas dos dormentes na imagem binária (em pixels) para unidades métricas $\left(V_{m}\right)$. A função utilizada para realizar essa transformação é apresentada na Equação 1.

$$
V_{m}=v_{\min }+\frac{v_{p x}}{1280}\left(v_{\max }-v_{\min }\right)
$$

onde $v_{p x}$ é a variável responsável por acomodar o valor em pixels dos pontos que devem ser redimensionados, $v_{\max } \mathrm{e}$ 


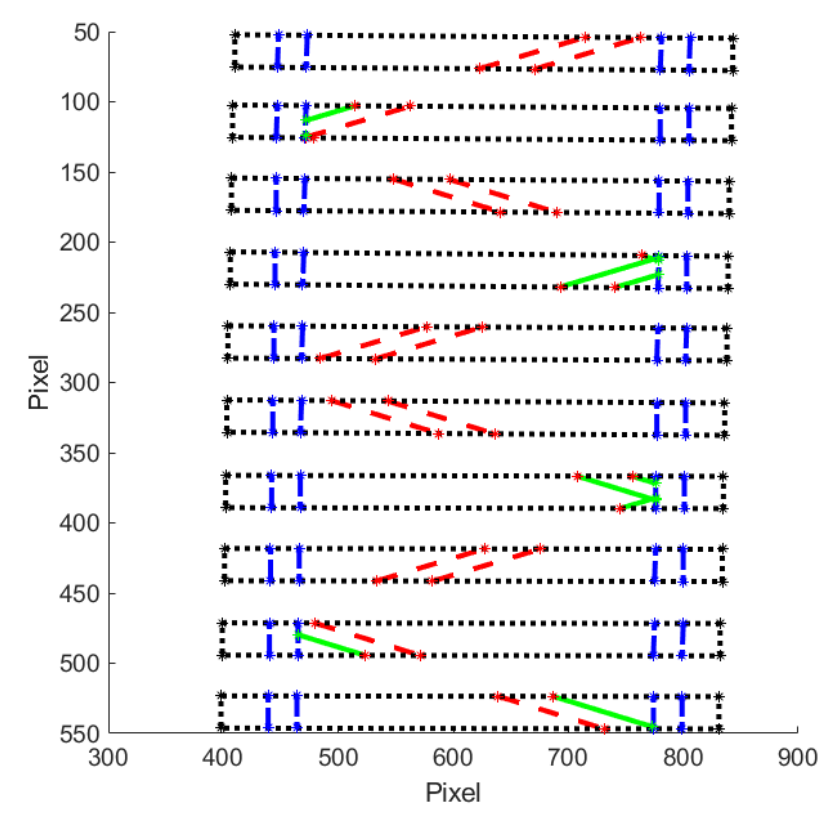

Figura 14. Dormentação com entalhes.

$v_{\text {min }}$ são valores máximo e mínimo no qual os pixels podem ser redimensionados.

A Figura 15 ilustra os pontos gerados após ser aplicada a Equação 1. Os pontos quadriculados pretos representam as quinas dos dormentes, os círculos são entalhes onde se encaixarão as longarinas e os asteriscos vermelhos são os entalhes nos quais os contraventamentos serão encaixados.

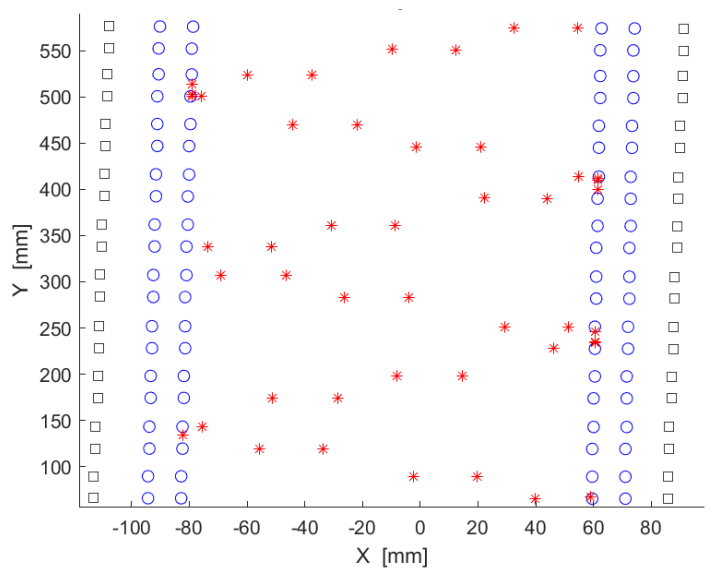

Figura 15. Pontos da dormentação com entalhes.

Com esses pontos é possível realizar a reconstrução 3D dos dormentes em qualquer software de geração de sólidos que aceite importação de dados em pontos. Neste trabalho a reconstrução foi realizada no software Autodesk Inventor. A Figura 16 ilustra o resultado da reconstrução 3D dos dormentes com os seus respectivos entalhes obtida a partir dos pontos após a aplicação da Equação 1.

\section{RESUlTADOS E DISCUSSÕES}

Este capítulo apresenta os resultados obtidos com a aplicação do método desenvolvido neste trabalho com os dados obtidos do modelo de maquete utilizado para valida-

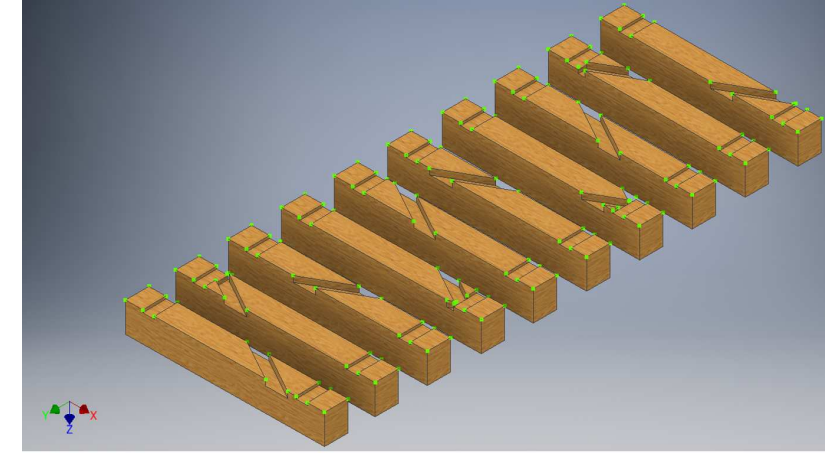

Figura 16. Sólido gerado a partir dos pontos obtidos pelo método proposto.

ção desta técnica. A Figura 17 ilustra o processamento executado pelo método nas três primeiras etapas, onde a Figura 17 (a) mostra a nuvem de pontos com préprocessamento de ruídos e recortes na zona de interesse. Essa nuvem de pontos em especial possui faixa mais abrangente a qual possibilita visualizar a superestrutura da via. A Figura 17 (b) mostra a nuvem de pontos das longarinas junto da reconstrução feita e a Figura 17 (c) mostra a nuvem de pontos da ponte com a dormentação gerada já com seus respectivos entalhes.

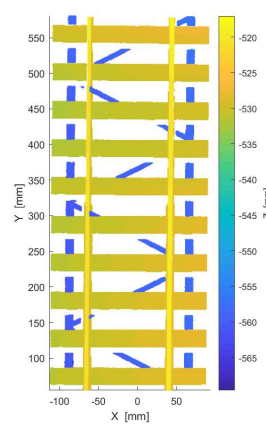

(a)

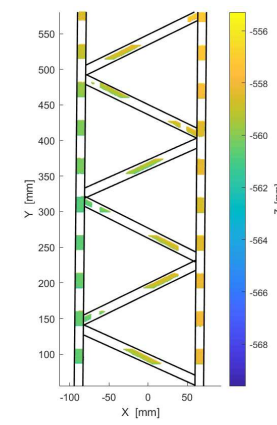

(b)

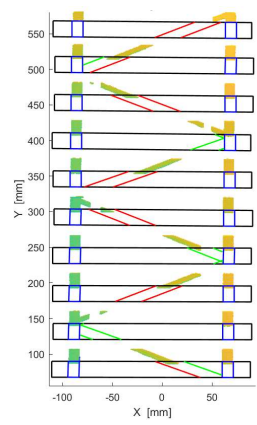

(c)
Figura 17. Nuvem de pontos, vista superior.

Com o auxílio do sólido gerado na Figura 16 e do projeto desenvolvido para impressão da maquete da ponte sem lastro, foi possível desenhá-los lado a lado utilizando a mesma plataforma de criação. Para facilitar a visualização dos entalhes foi realizado o corte dos dormentes rente a estrutura da ponte. A Figura 18 (a) mostra o desenho da maquete e a Figura 18 (b) a reconstrução do sólido realizada a partir do método proposto com um corte feito rente as estrutura da ponte.

\subsection{Erro de Reconstrução}

O cálculo do erro percentual de reconstrução foi realizado tendo os valores do desenho da maquete como base e pode ser visto na Equação 2.

$$
E_{r}=\frac{\left|A_{p m}-A_{p r}\right|}{A_{p m}} * 100,
$$

onde $E_{r}$ é o erro percentual, $A_{p m}$ é a área de superfície na face de entalhe do dormente da maquete e $A_{p r}$ é a área de superfície na face de entalhe do dormente reconstruído. 


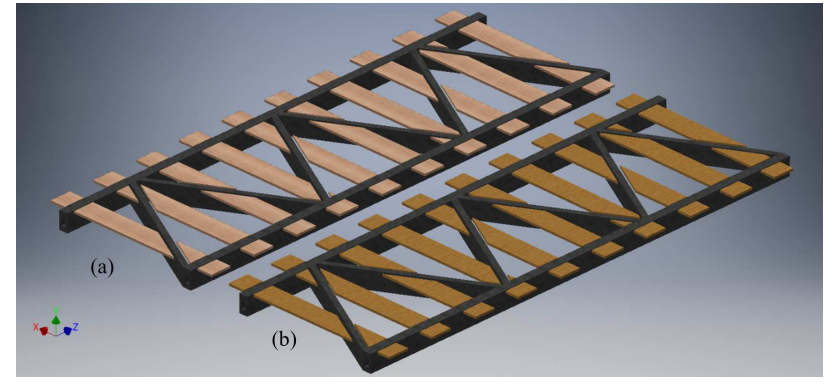

Figura 18. Desenho da maquete (a) e sólido gerado (b) com corte rente à estrutura da ponte.

O erro percentual foi calculado para cada dormente separadamente. O primeiro cálculo foi feito abordando somente as áreas sem entalhes, como ilustrado na Figura 19 (a), onde as áreas sem entalhes estão em destaque na cor azul. Também foi encontrado o erro percentual das áreas com entalhes ilustrado na Figura 19 (b). E por fim o percentual da área total com e sem entalhe ilustrado na Figura 19 (c).

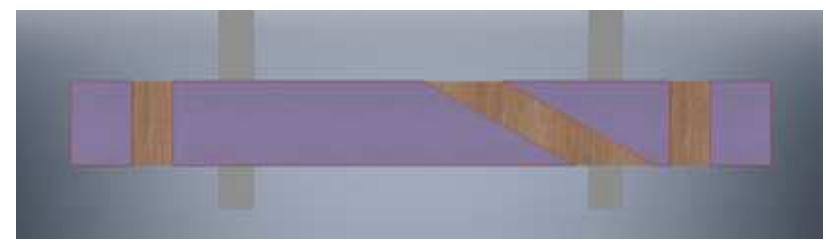

(a)

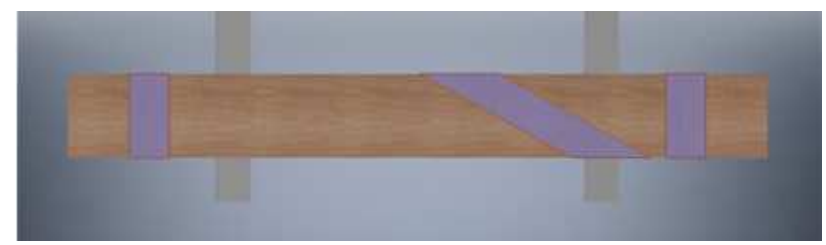

(b)

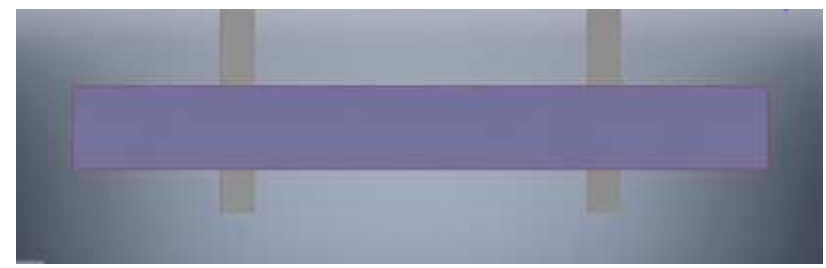

(c)

Figura 19. Destaque das áreas utilizadas para cálculo do erro percentual.

A Tabela 1 apresenta o erro percentual de reconstrução de cada um dos 10 dormentes existentes na maquete utilizada para este trabalho. Essa tabela apossui em suas colunas o erro percentual sem entalhe, com entalhe e da área total do dormente.

Com base nesses valores é possível chegar à conclusão que a reconstrução realizada possui alta confiabilidade, com erro percentual médio total de 1,5\%. Este resultado se mostra bastante promissor e encorajador para desenvolver a próxima etapa deste projeto, a qual consiste em desenvolver uma plataforma móvel autônoma dotada de sensores de perfil laser e encoders para realizar a medida de uma ponte em tamanho real. A Figura 20 apresenta o projeto
Tabela 1. Erro Percentual.

\begin{tabular}{|c|c|c|c|}
\hline Dormente & $\begin{array}{c}\text { Erro da área } \\
\text { sem Entalhe }\end{array}$ & $\begin{array}{c}\text { Erro da área } \\
\text { com Entalhe }\end{array}$ & Erro Total \\
\hline 1 & $1,387 \%$ & $2,013 \%$ & $1,529 \%$ \\
\hline 2 & $2,128 \%$ & $2,172 \%$ & $1,302 \%$ \\
\hline 3 & $1,534 \%$ & $1,513 \%$ & $1,529 \%$ \\
\hline 4 & $1,223 \%$ & $2,815 \%$ & $1,529 \%$ \\
\hline 5 & $0,945 \%$ & $2,513 \%$ & $1,302 \%$ \\
\hline 6 & $1,534 \%$ & $1,513 \%$ & $1,529 \%$ \\
\hline 7 & $1,546 \%$ & $1,453 \%$ & $1,529 \%$ \\
\hline 8 & $1,534 \%$ & $1,513 \%$ & $1,529 \%$ \\
\hline 9 & $1,705 \%$ & $0,873 \%$ & $1,529 \%$ \\
\hline 10 & $1,491 \%$ & $1,660 \%$ & $1,529 \%$ \\
\hline
\end{tabular}

da estrutura motorizada que será utilizada na realização dos experimentos.

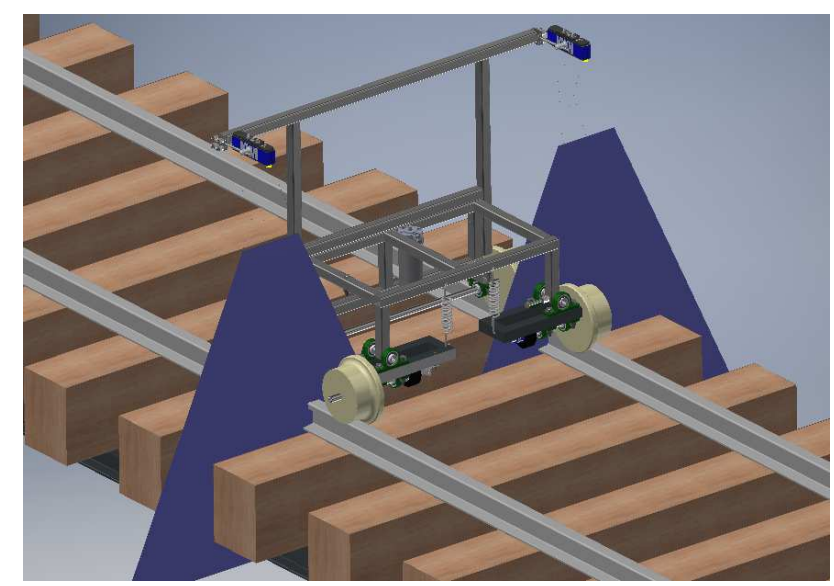

Figura 20. Estrutura do veículo autônomo que será utilizado para realizar os experimentos em pontes sem lastro.

\section{CONCLUSÕES E TRABALHOS FUTUROS}

Este artigo apresentou um método para estimar os perfis dos dormentes de uma ponte sem lastro utilizando dados obtidos de um sensor perfil laser. As informações capturadas pelo sistema sensorial foram inicialmente transformadas em uma nuvem de pontos 3D que apresentam a estrutura da ponte sem lastro escaneada. A partir dessa nuvem, foi criada uma imagem binária a qual é branca nas regiões de interesse (longarinas e contraventamentos) e preta nas regiões que não são relevantes.

O método implementado foi capaz de reconstruir a estrutura da ponte sem lastro em escala e também estimar os entalhes que precisam ser feitos nos dormentes para uma eventual substituição durante a manutenção da ponte. Os resultados obtidos apresentaram um erro percentual médio total de aproximadamente $1,5 \%$.

Como trabalhos futuros serão realizados experimentos em pontes reais utilizando uma estrutura motorizada dotada de sensores perfil laser, capaz de se locomover autonomamente sobre as pontes e capturar os dados necessários para a reconstrução $3 \mathrm{D}$ do ambiente.

Com a implementação desse trabalho o tempo de interdição da via será reduzido por conta de grande parte do trabalho de marcenaria ser realizado fora da ponte diminuindo o tempo de exposição do especialista. 


\section{AGRADECIMENTOS}

Os autores gostariam de agradecer ao Instituto Federal do Espírito Santo pelo apoio para o desenvolvimento deste trabalho.

\section{REFERÊNCIAS}

Bosché, F. (2010). Automated recognition of 3D cad model objects in laser scans and calculation of asbuilt dimensions for dimensional compliance control in construction. Advanced Engineering Informatics, 24, 107-118.

Brochado, M.R. and Pires, F.P. (2007). Introdução da tecnologia laserail na manutenção da via permanente metro-ferroviária. In XXVII Encontro Nacional de Engenharia de Produção - ENEGEP $200 \%$.

Campos, J.J.L. (2015). Análise de dormente polimérico reciclado como solução financeira viável para substituição de dormente de madeira dos AMV's da via permanente. In XXI Semana de Tecnologia Metroferroviária - AEAMESP 2015.

Franca, A.S. (2017). Identificação e Monitoramento de Dormentes de Ferrovias Usando Processamento de Imagens. Master's thesis, Universidade Federal do Espírito Santo - UFES.

Harrison, A. and Newman, P. (2008). High quality 3d laser ranging under general vehicle motion. In 2008 IEEE International Conference on Robotics and Automation, 7-12. doi:10.1109/ROBOT.2008.4543179.

Klimentjew, D., Arli, M., and Zhang, J. (2009). 3d scene reconstruction based on a moving $2 \mathrm{~d}$ laser range finder for service-robots. In 2009 IEEE International Conference on Robotics and Biomimetics (ROBIO), 11291134. doi:10.1109/ROBIO.2009.5420757.

Llamazares, A., Molinos, E.J., Ocaña, M., Bergasa, L.M., Hernández, N., and Herranz, F. (2012). 3d map building using a $2 \mathrm{~d}$ laser scanner. In Proceedings of the 13th International Conference on Computer Aided Systems Theory - Volume Part II, EUROCAST'11, 412-419. Springer-Verlag, Berlin, Heidelberg.

Lourenço, M.K. (2014). Elaboração de projeto para o desenvolvimento de material didático para o ensino de engenharia ferroviária e metroviária com foco em via permanente. Technical report, Universidade Federal de Santa Catarina - UFSC.

Lubowiecka, I., Armesto, J., Arias, P., and Lorenzo, H. (2009). Historic bridge modelling using laser scanning, ground penetrating radar and finite element methods in the context of structural dynamics. Engineering Structures, 31, 2667-2676.

Rabah, M., Elhattab, A., and Fayad, A. (2013). Automatic concrete cracks detection and mapping of terrestrial laser scan data. Journal of Astronomy and Geophysics, 2, 250-255.

Ren, S., Gu, S., Xu, G., Gao, Z., and Feng, Q. (2011). A new track inspection car based on a laser camera system. Chinese Optics Letters, 9(3), 31202-31205. doi: 10.3788/COL20110903.31202.

Silva, L.F.M. (2002). Fundamentos Teórico-Experimentais da Mecânica dos Pavimentos Ferroviários e Esboço de um Sistema de Gerência Aplicado à Manutenção da Via Permanente. Ph.D. thesis, Universidade Federal do Rio de Janeiro - UFRJ.
Vale (2009). Manual técnico da via permanente. Technical report, Vale.

Yang, B. and Fang, L. (2014). Automated extraction of 3-d railway tracks from mobile laser scanning point clouds. IEEE Journal of Selected Topics in Applied Earth Observations and Remote Sensing, 7(12), 47504761. doi:10.1109/JSTARS.2014.2312378. 